\title{
Magnetic anisotropy in antiferromagnetic hexagonal MnTe
}

\author{
D. Kriegner, ${ }^{1,2,{ }^{*}}$ H. Reichlova, ${ }^{1}$ J. Grenzer, ${ }^{3}$ W. Schmidt ${ }^{4}$ E. Ressouche, ${ }^{5}$ J. Godinho, ${ }^{1}$ T. Wagner, ${ }^{6}$ S. Y. Martin,${ }^{6,7}$ \\ A. B. Shick, ${ }^{8}$ V. V. Volobuev, $,{ }^{9}, 10$ G. Springholz, ${ }^{9}$ V. Holý, ${ }^{2}$ J. Wunderlich, ${ }^{6}$ T. Jungwirth, ${ }^{1,11}$ and K. Výborný ${ }^{1}$ \\ ${ }^{1}$ Institute of Physics, Academy of Science of the Czech Republic, Cukrovarnická 10, 16200 Praha 6, Czech Republic \\ ${ }^{2}$ Charles University in Prague, Ke Karlovu 3, 12116 Praha 2, Czech Republic \\ ${ }^{3}$ Helmholtz-Zentrum Dresden-Rossendorf, Institute of Ion Beam Physics and Materials Research, \\ Bautzner Landstrasse 400, 01328 Dresden, Germany \\ ${ }^{4}$ Jülich Centre for Neutron Science JCNS, Forschungszentrum Jülich GmbH, Outstation at ILL, \\ CS 20156, 71 avenue des Martyrs, F-38042 Grenoble, France \\ ${ }^{5}$ Université Grenoble Alpes, CEA, INAC-MEM, 38000 Grenoble, France \\ ${ }^{6}$ Hitachi Cambridge Laboratory, Cambridge CB3 OHE, United Kingdom \\ ${ }^{7}$ Université Grenoble Alpes, CNRS, CEA, Grenoble INP, INAC-Spintec, 38000 Grenoble, France \\ ${ }^{8}$ Institute of Physics, Academy of Science of the Czech Republic, Na Slovance 1999/2, 18221 Praha 8, Czech Republic \\ ${ }^{9}$ Institute of Semiconductor and Solid State Physics, Johannes Kepler University Linz, Altenbergerstr. 69, 4040 Linz, Austria \\ ${ }^{10}$ National Technical University, "Kharkiv Polytechnic Institute," 61002 Kharkiv, Ukraine \\ ${ }^{11}$ School of Physics and Astronomy, University of Nottingham, Nottingham NG7 2RD, United Kingdom
}

(Received 27 October 2017; published 13 December 2017)

\begin{abstract}
Antiferromagnetic hexagonal MnTe is a promising material for spintronic devices relying on the control of antiferromagnetic domain orientations. Here we report on neutron diffraction, magnetotransport, and magnetometry experiments on semiconducting epitaxial MnTe thin films together with density functional theory (DFT) calculations of the magnetic anisotropies. The easy axes of the magnetic moments within the hexagonal basal plane are determined to be along $\langle 1 \overline{1} 00\rangle$ directions. The spin-flop transition and concomitant repopulation of domains in strong magnetic fields is observed. Using epitaxially induced strain the onset of the spin-flop transition changes from $\sim 2$ to $\sim 0.5 \mathrm{~T}$ for films grown on $\mathrm{InP}$ and $\mathrm{SrF}_{2}$ substrates, respectively.
\end{abstract}

DOI: 10.1103/PhysRevB.96.214418

\section{INTRODUCTION}

Antiferromagnets (AFMs) have recently attracted considerable attention in the context of spintronic devices [1,2] not only as passive components (e.g., pinning layers in magnetic tunnel junctions) but also directly as a medium to store information [3]. One of the key requirements for magnetically ordered materials to provide device functionality is the possibility to manipulate the magnetic moments. Albeit not straightforward for AFMs (at least in comparison to ferromagnets), this turns out to be possible in several ways [4-7]. For example, spin-orbit torques either induced by interfaces [5] or by the inverse spin galvanic effect inside the antiferromagnet [6] can be used to manipulate antiferromagnetic states. Further, field cooling through the Néel temperature $[4,7]$ or high magnetic fields applied in the antiferromagnetic state [7] were shown to manipulate the domain population. For this purpose, as well as for detection and stability of ordered magnetic states, magnetic anisotropies (MAs) have to be considered carefully, which is the aim of this work.

One of the main advantages of using AFMs instead of ferromagnets in spintronics is the availability of a broad variety of intrinsic antiferromagnetic semiconductors. They allow for merging the vast amount of spintronic effects with electrical controlled transport properties of a semiconductor. Among them, hexagonal manganese telluride (MnTe) had already been extensively studied well before the advent of spintronics, in nineteen sixties and nineteen seventies, for its

*dominik.kriegner@gmail.com optical [8] or magnetical [9] properties. It has a relatively high Néel temperature $\left(T_{\mathrm{N}}\right)$ of $310 \mathrm{~K}[10]$ and a moderate band gap of 1.27 to $1.46 \mathrm{eV}[7,11]$. Typically, MnTe is a $p$-type semiconductor but intentional doping with sodium or chromium can tune the resistivity over a wide range [12]. The magnetic structure of MnTe was determined from neutron diffraction and consists of ferromagnetic hexagonal Mn planes which are antiferromagnetically coupled along the $c$ axis [13-16]. The determination of magnetic moment of $\mathrm{Mn}$ atoms was a subject of several experimental works (see the summary in Ref. [13]), the scatter being relatively large (from values close to $5 \mu_{B}$ all the way down to almost $4 \mu_{B}$ ) and moreover, the easy axis was not determined. Neither spin-wave measurements by inelastic neutron diffraction could resolve the MA within the hexagonal $c$ plane [17] although this MA is certainly present as implied by torque magnetometry [9] and recent anisotropic magnetoresistance measurements [7]. Both measurements have shown that three distinct orientations of domains exist within the hexagonal plane. The possibility to change the domain population almost continuously [7] also affords memristive behavior [18-20] to MnTe-based devices.

Although bulk materials were explored first, the attention turned later to thin layers grown on various substrates, leading to the discovery of a new MnTe phase. Apart from the hexagonal phase [NiAs-type, $\alpha$-phase, $\mathrm{P}_{3} / \mathrm{mmc}$ (194), Fig. 1(a)], which is stable in bulk form, zinc-blende MnTe films with a larger optical band gap and much lower Néel temperature [21] were found to be epitaxially stabilized on GaAs substrates with and without a CdTe buffer layer [22-24]. Epitaxial thin film growth of the hexagonal $\alpha$-MnTe phase, which is the topic of this work, was demonstrated on single crystalline $\mathrm{SrF}_{2}(111)$, 


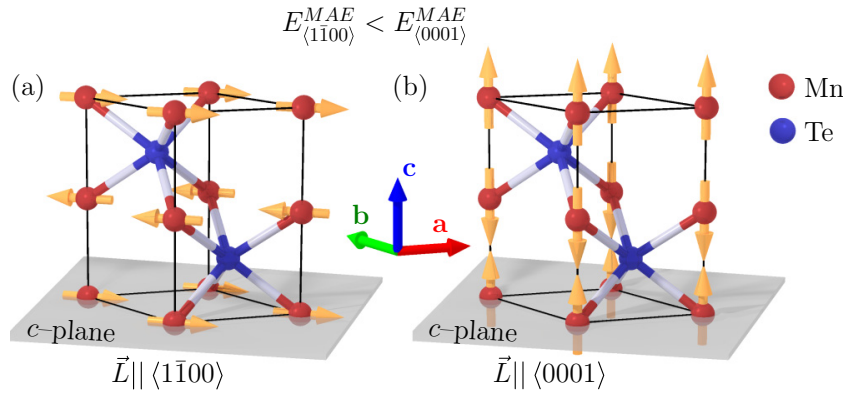

FIG. 1. Sketch of the atomic and possible magnetic structures of antiferromagnetic hexagonal MnTe. (a) In-plane/c plane (ground state) and (b) out-of-plane/c-axis (hard axis) orientation of the magnetic moments of Mn with the Néel vector $\vec{L}$ along $\langle 1 \overline{1} 00\rangle$ and $\langle 0001\rangle$ are shown. The hexagonal basal plane, i.e., the $c$ plane is indicated by a gray plane, while red, green, and blue arrows show the directions of the unit cell axes.

$\operatorname{InP}(111)$ [7], and $\mathrm{Al}_{2} \mathrm{O}_{3}(0001)[16,25,26]$ substrates as well as on amorphous $\mathrm{Si}(111) / \mathrm{SiO}_{2}$ [27]. Due to lattice and thermal expansion coefficient mismatch between $\alpha$-MnTe and the substrates, films will experience strain that may affect the magnetic properties such as MAs. For example [28], the dilute magnetic semiconductor (Ga,Mn)As is known to have an in-plane MA under compressive strain and an out-of-plane MA for tensile strain under suitable conditions. Here, we study the MAs in MnTe on different substrates, which cause different strain states. The knowledge of the easy axis directions is crucial for transport phenomena modeling, which has so far relied only on assumptions [7]. As far as the easy axis directions are concerned, we confirm these assumptions using DFT $+U$ calculations combined with experiments. Using magnetotransport, magnetometry, and neutron diffraction, we determine the easy axes to be along $\langle 1 \overline{1} 00\rangle$ and show in what respect MAs are sensitive to epitaxy-induced strain.

The paper is organized as follows. After introduction of the results of DFT $+U$ calculations in Sec. II, we describe our samples structure and basic magnetometry characterization in Sec. III. Section IV presents our neutron diffraction experiments and Sec. V complementary magneto-transport studies. Further magnetometry experiments determining the spin-flop field are presented in Sec. VI. Finally, we conclude in Sec. VII.

\section{MAGNETIC ANISOTROPY CALCULATIONS}

The magnetic anisotropy energy (MAE) in antiferromagnets comprises two main contributions: the dipole term and the magnetocrystalline anisotropy (MCA). In order to calculate the latter, we use the relativistic version of the rotationally invariant $\mathrm{DFT}+U$ method [29], which takes into account spin-orbit coupling, and nondiagonal in spin contributions into the occupation matrix. The full-potential linearized augmented plane-wave (FLAPW) [30] basis is used in the self-consistent total energy calculations. We use $U=4 \mathrm{eV}$ and $J=0.97 \mathrm{eV}$ parameters taken from a similar compound of manganese [31].

The dipole term is a classical contribution from dipoledipole interaction of localized magnetic moments [32]. For coherent rotations of the two AFM sublattices which strictly maintain their antiparallel alignment, e.g., one that interpolates between the two magnetic configurations shown in Fig. 1, the dipole term depends in general on the rotation angle. This dependence is absent for cubic crystals but present in $\mathrm{MnTe}$ since the crystal symmetry of the NiAs structure is lower. This causes the energy of the dipole-dipole interaction of the structure in Fig. 1(b), with magnetic moments aligned along the $c$-axis, to be higher than that of any structure with magnetic moments oriented in the hexagonal basal plane ( $c$ plane), e.g., Fig. 1(a).

For lattice constants $a=0.4134 \mathrm{~nm}$ and $c=0.6652 \mathrm{~nm}$ [experimentally determined at $5 \mathrm{~K} / \mathrm{see}$ Sec. III, Figs. 3(a) and 3(b)], we obtain that Mn atoms carry the magnetic moments of $4.27 \mu_{B}$ (spin $M_{S}=4.25 \mu_{B}$ plus orbital $M_{L}=0.02 \mu_{B}$ magnetic moments). The energy difference of the two different configurations shown in Fig. 1 from the dipole term $E^{\text {dipole }}$ is calculated to be $0.135 \mathrm{meV}$ per unit cell, favoring the alignment in the $c$ plane. This contribution to MAE is only weakly dependent on strain or relevant lattice distortions and gives no anisotropy within the $c$ plane.

The DFT $+U$ calculations of the MCA are much more involved but, rather generally, a clear picture emerges of moderately large out-of-plane anisotropy and small anisotropies within the $c$ plane. For the lattice constants quoted above, an energy difference between configurations in Figs. 1(a) and 1(b) of $0.11 \mathrm{meV}$ per unit cell is calculated again favoring the alignment in the $c$ plane. The anisotropy within the $c$ plane, defined as the energy difference between the magnetic structure in Fig. 1(a) and one with magnetic moments rotated by $90^{\circ}$ in the $c$ plane, is small and at the edge of the accuracy $(10 \mu \mathrm{eV})$ of the calculation in this particular case.

To model actual conditions in our experiments, we perform zero-temperature calculations of $E^{\mathrm{MCA}}$ for various choices of lattice constants (see Table I). Adding the MCA to the dipole term, we can conclude that (a) the out-of-plane MAE is typically between 0.2 and $0.3 \mathrm{meV}$ per unit cell (two formula units), favoring the moments within the $c$ plane, and (b) the anisotropy within the $c$ plane is typically an order of magnitude smaller. For calculations under changing $c / a$ ratio shown in Table I, the MAE within the $c$ plane is always smaller than the out-of-plane MAE (even for the extreme choice of lattice constants with $c=0.689 \mathrm{~nm}$, see Table I, the latter is greater than $0.1 \mathrm{meV}$ per unit cell), the MAE within the $c$ plane exhibits no clear trend upon unit cell deformation and it even changes sign. In order to unambiguously determine anisotropies within the $c$ planes, it is therefore advisable to resort to experiments.

TABLE I. The total MAE, $E^{\text {dipole }}+E^{\mathrm{MCA}}$ in meV per unit cell for different lattice parameters. The Néel vector directions with respect to the crystal are given as subscript of the energies, showing the preferential magnetic moment orientation in the $c$ plane.

\begin{tabular}{lrllllr}
\hline \hline$a(\mathrm{~nm})$ & 0.408 & 0.411 & 0.414 & 0.417 & 0.408 & 0.408 \\
$c(\mathrm{~nm})$ & 0.670 & 0.670 & 0.670 & 0.670 & 0.650 & 0.689 \\
$E_{[0001]}-E_{[11 \overline{1} 0]}$ & 0.20 & 0.24 & 0.23 & 0.22 & 0.28 & 0.12 \\
$E_{[1 \overline{1} 00]}-E_{[11 \overline{1} 0]}$ & -0.01 & 0.03 & 0.01 & 0.04 & 0.05 & -0.01 \\
\hline \hline
\end{tabular}




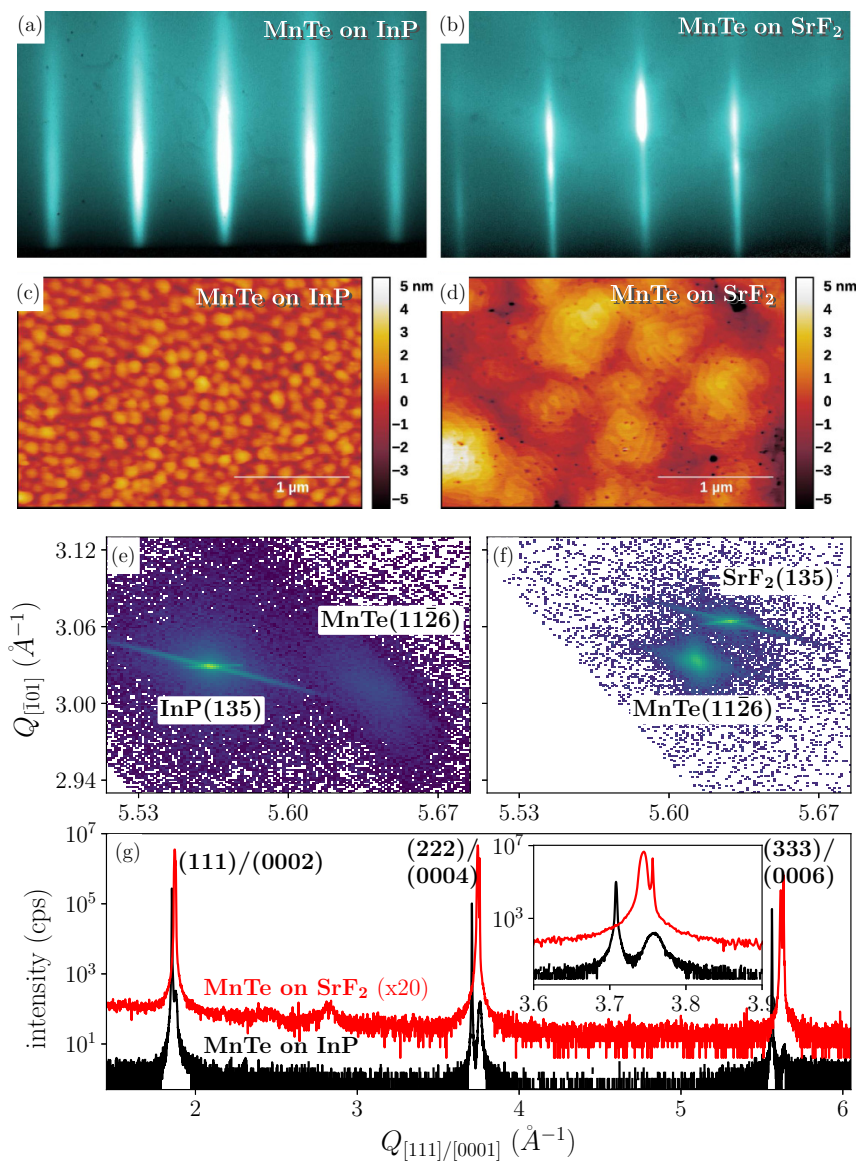

FIG. 2. Reflection high-energy electron diffraction (RHEED) patterns of 50-nm and 2500-nm thick epitaxial MnTe films on InP(111)A (a) and $\mathrm{SrF}_{2}(111)$ (b), respectively, recorded along the [1110] zone axis. Atomic force microscope images for MnTe films on InP(111)A and $\mathrm{SrF}_{2}(111)$ are shown in (c) and (d). X-ray diffraction reciprocal space maps around the (135) substrate Bragg reflection of the samples are shown in (c) and (d). Due to the epitaxial relationship, the (1126) Bragg reflection of MnTe is seen close to the substrate peaks. (e) shows the symmetric radial scan for MnTe on InP (black) and $\mathrm{MnTe}$ on $\mathrm{SrF}_{2}$ (red). For clarity, the trace of $\mathrm{MnTe}$ on $\mathrm{SrF}_{2}$ was scaled by a factor of 20. The inset shows a zoom around the (222) substrate Bragg peaks and the broader (0004) Bragg peaks of the MnTe epilayer. Data in (c)-(e) are recorded at room temperature where due to different strain states the Bragg peak position of the films is slightly different for the two used substrate materials.

\section{SAMPLE STRUCTURE AND MAGNETOMETRY}

\section{A. Sample growth and structure}

Single crystalline hexagonal MnTe epilayers were grown by molecular beam epitaxy on single crystalline $\mathrm{SrF}_{2}(111)$ and In-terminated $\mathrm{InP}(111) \mathrm{A}$ substrates using elemental $\mathrm{Mn}$ and $\mathrm{Te}$ sources. Both types of substrates have a cubic structure $\left(\mathrm{CaF}_{2}\right.$ and zinc blende, respectively) with lattice parameters of $a_{0}=$ $0.57996 \mathrm{~nm}$ for $\mathrm{SrF}_{2}$ and $0.58687 \mathrm{~nm}$ for InP at room temperature. However, the respective surface lattice constants of the (111) surfaces $\left(a_{0} / \sqrt{2}\right)$ of 0.410 and $0.415 \mathrm{~nm}$ are very close to the hexagonal $a$ lattice constant of $\alpha-\operatorname{MnTe}(a=0.414 \mathrm{~nm}$ and $c=0.671 \mathrm{~nm}[33]$ ). Thus both types of substrates are very well suited for MnTe growth with a lattice mismatch of less than $1 \%$ in both cases, which resulted in single crystalline films with epitaxial interface between film and substrate [7]. Indeed two-dimensional growth of $\alpha-\mathrm{MnTe}$ is achieved at the used substrate temperatures in the range of $370{ }^{\circ} \mathrm{C}-450{ }^{\circ} \mathrm{C}$ as indicated by the streaked reflection high-energy electron diffraction (RHEED) patterns observed during growth as shown in Figs. 2(a) and 2(b). The resulting film morphology can be seen in the atomic force microscopy images in Figs. 2(c) and 2(d). In both cases, a root mean square roughness of $\sim 1 \mathrm{~nm}$ is observed. From $\mathrm{x}$-ray diffraction measurements, shown in Figs. 2(e)-2(g), we find that the MnTe layers grow with the $c$ axis perpendicular to the (111) substrate surfaces with an epitaxial relationship of (0001)[1 100$]_{\mathrm{MnTe}}||(111)[11 \overline{2}]_{\mathrm{SrF} / \mathrm{InP}}$, which corresponds to hexagon-on-hexagon-like matching. Thus we refer to the $c$ axis as out-of-plane direction and all perpendicular directions including $\langle 10 \overline{1} 0\rangle$ and $\langle 11 \overline{2} 0\rangle$, i.e., within the $c$ plane, are called in-plane directions. Note that we use the Miller indices $h k l$ to denote cubic and Bravais indices $h k i l$ with $i=-h-k$ to denote the hexagonal lattice points/directions and that crystallographic directions refer to the corresponding real space directions. In addition, no traces of secondary MnTe phases are found in the wide range diffraction scans in Fig. 2(g).

From reciprocal space maps as shown in Figs. 2(e) and 2(f), the in-plane and out-of-plane lattice constants of the epilayers $a$ and $c$ were determined. For all MnTe films on $\mathrm{SrF}_{2}$ (111) thicker than $50 \mathrm{~nm}$, we generally find that the in-plane lattice constant is very close the MnTe bulk value indicating that the films are nearly fully relaxed. On the contrary, the films on InP (111) exhibit an in-plane lattice constant larger than bulk MnTe in spite of the closer lattice matching. This is explained by the different thermal expansion coefficients of the film and the substrate. Above room temperature, the thermal expansion coefficient of $\mathrm{SrF}_{2}$ is $2.0 \times 10^{-5} \mathrm{~K}^{-1}$ (Ref. [34]), which is only $20 \%$ larger than the value of $1.62 \times 10^{-5} \mathrm{~K}^{-1}$ (Ref. [35]) of MnTe, for which reason the cooling of the sample from the growth temperature to room temperature does not induce a significant thermal strain in the films due to nearly the same thermal contraction. Conversely, the thermal coefficient of InP of $0.5 \times 10^{-5} \mathrm{~K}^{-1}$ (Ref. [36]) is about three times smaller than that of MnTe and therefore, the cooling to room temperature induces a significant tensile strain in the epilayers exceeding $0.5 \%$. Thus MnTe films on InP are subject to biaxial tensile strain whereas those on $\mathrm{SrF}_{2}$ are nearly fully relaxed. For micrometer-thick MnTe films on InP, the large thermal expansion mismatch stress leads to the formation of microcracks in the films as well as partial delamination. For this reason, only thick films on $\mathrm{SrF}_{2}$ were used for our neutron diffraction investigations. For the investigations of the magnetic anisotropy of the films on InP, the film thickness was restricted to $50 \mathrm{~nm}$ and therefore magnetotransport measurements instead of neutron diffraction were used.

The different thermal expansion of the layers and substrates will also modify the strain state of the MnTe films at low temperatures (neutron diffraction and magnetotransport investigations are performed at liquid Helium temperatures). Therefore we measured in addition the temperature dependence of the lattice parameters by x-ray diffraction as shown in Figs. 3(a) and 3(b). When cooled from room temperature, 

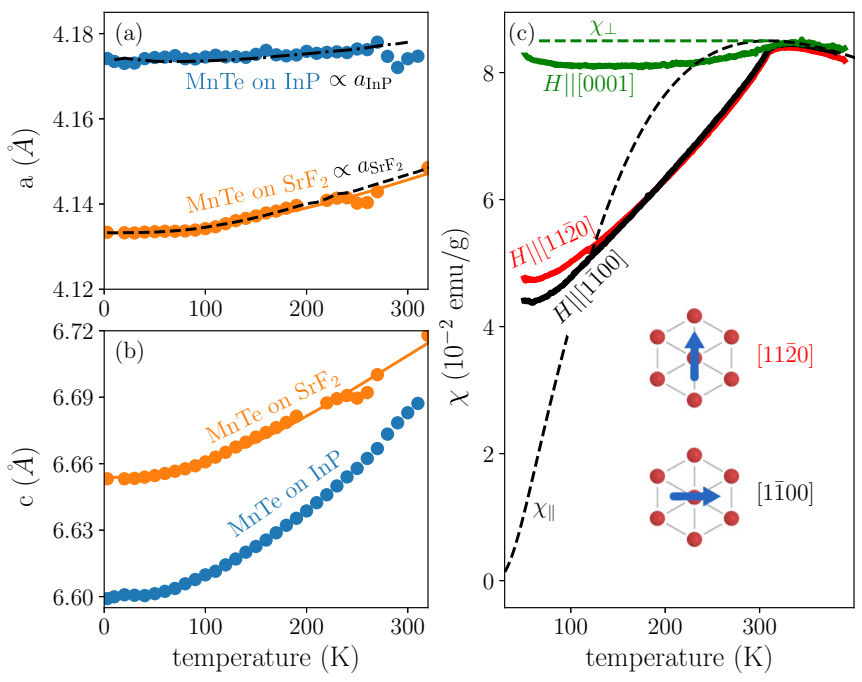

FIG. 3. (a), (b) Temperature-dependent $a$ and $c$ lattice parameters of MnTe grown on two different substrates. The dashed (dash-dot) line in (a) represents the measured temperature dependence of the $\mathrm{SrF}_{2}$ (InP) substrate lattice parameter scaled by $\sqrt{2} \times 1.01(\sqrt{2} \times 1.007)$. Note that the solid lines shown for the case of $\mathrm{MnTe}$ on $\mathrm{SrF}_{2}$ are guides to the eye since measurements around room temperature are hampered by overlapping signals of the thin film and substrate. (c) Temperaturedependent susceptibility of $2.5-\mu \mathrm{m}$ MnTe on $\mathrm{SrF}_{2}$ measured for a magnetic field applied in different directions. The diamagnetic contribution of the substrate was subtracted. Dashed lines show the mean-field susceptibility of a collinear uniaxial antiferromagnet for the cases when the field is perpendicular $\left(\chi_{\perp} /\right.$ green $)$ and parallel $\left(\chi_{\|} /\right.$black $)$to the easy axis. Insets indicate the directions of the magnetic field with respect to the crystal within the $c$ plane.

the in-plane lattice constant $a$ of both films on $\mathrm{SrF}_{2}$ and InP basically follows the change of the scaled substrate lattice parameter, which is also plotted in Fig. 3(a) by the dashed and dash-dotted lines. This means that the in-plane strain of the MnTe film on InP even increases, whereas only small changes occur on $\mathrm{SrF}_{2}$. Note that the scaling of the substrate surface lattice parameters by around $1 \%$ indicates the relaxation of the epitaxial films during growth. At liquid He temperatures, the in-plane lattice constant of the MnTe films differs by as much as $1.0 \%$ for the different substrates. This leads also to a different evolution of the out-of-plane $c$-axis lattice constant of the films on InP and $\mathrm{SrF}_{2}$ as shown in Fig. 3(b). Our theoretical calculations in Table I indicate that while the out-of-plane MAE remains dominant upon such variations of strain, the in-plane MAE may change substantially, potentially even to the point that the direction of the easy axis (within the basal plane) changes.

\section{B. Magnetometry}

One possible way of determining the natural orientation of magnetic moments, i.e., the easy axis direction, is the measurement of the temperature-dependent susceptibility $\chi$ shown in Fig. 3(c). Very early on [37], it has been recognized that while $\chi_{\|}(T)$ (magnetic field applied parallel to magnetic moments) for a uniaxial antiferromagnet drops to zero as $T \rightarrow 0$, a magnetic field applied in (any) perpendicular

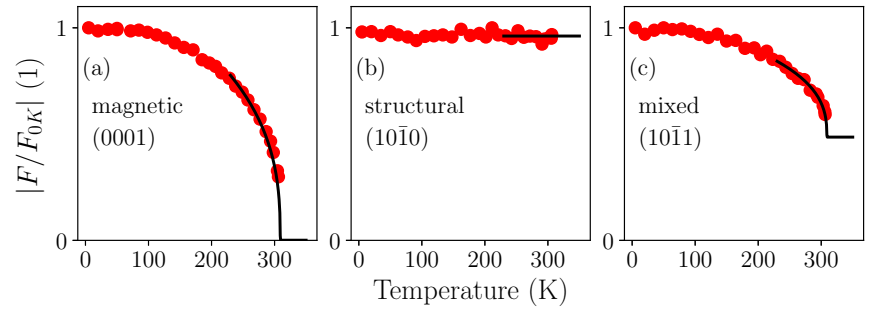

FIG. 4. Neutron diffraction structure factors measured vs temperature for the (a) magnetic (0001), (b) structural (1010), and (c) mixed structural and magnetic Bragg peak (1011). Black lines show the behavior close to the Néel temperature described by the equation $A_{\text {mag }}\left(T_{\mathrm{N}}-T\right)^{c}+A_{\text {struct }}$, where $A_{i}$ denotes the amplitude of magnetic and structural contribution and $T$ the measurement temperature. The Néel temperature $T_{\mathrm{N}}=309 \mathrm{~K}$ and the critical exponent $c=0.37$ corresponds to the Heisenberg model [38].

direction gives a constant $\chi_{\perp}(T)=\chi_{0}$ for $T<T_{\mathrm{N}}$. An explicit form of $\chi_{0}$ as well as $\chi_{\|}(T)$ based on Weiss theory can be found in Ref. [2]. We show this mean-field theory result for $S=5 / 2$ and scaled to the experimental data in Fig. 3(c) as dashed lines. Experimental data for $H \|[0001]$ therefore confirm that magnetic moments lie in the basal plane. On the other hand, since neither of the other two curves for $H \|[11 \overline{2} 0]$ and $H \|[1 \overline{1} 00]$ approaches zero for low temperatures, we conclude that there is not one single easy axis (or in other words, the sample is not uniaxial and therefore not in a single domain state). The small difference between these two curves suggests that the anisotropy within the $c$ planes is small.

\section{NEUTRON DIFFRACTION INVESTIGATIONS}

Experiments at the CEA-CRG thermal neutron diffractometer D23 at Institut Laue-Langevin in Grenoble, France allowed us to determine the easy axis in MnTe layers grown on $\mathrm{SrF}_{2}$. A monochromatic beam of neutrons with a wavelength of 0.127 nm was generated by a $\mathrm{Cu}$ (200) monochromator. The sample was mounted in a rotatable cryomagnet with temperature range of 5 to $305 \mathrm{~K}$ and magnetic fields up to $6 \mathrm{~T}$ along the sample rotation axis. The diffraction geometry with two orthogonal rotation axes of the detector allowed us to access several MnTe Bragg peaks sufficiently separated from those of the substrate. In Fig. 4, we show the intensity of selected diffraction peaks as a function of temperature. Since nonpolarized neutrons were used, the magnetic diffraction intensity depends solely on the relative orientation of the magnetic moments and the momentum transfer, and is at maximum when the magnetic moment is perpendicular to the momentum transfer. The shown variation of the (0001) diffraction peak [Fig. 4(a)], which is structurally forbidden in the paramagnetic phase, indicates that the magnetic moment within the $c$ plane has a significant value. In contrast to that, a peak with momentum transfer within the $c$ plane [see (10 $\overline{1} 0)$ in Fig. 4(b)] shows no magnetic contribution and therefore its intensity is virtually independent of temperature. The variation of the structure factors close to Néel temperature can be described by the critical behavior of the Heisenberg model with exponent $c=0.37$ [38] and is shown as solid line in Fig. 4. The ratio of intensities of the purely structural and magnetic Bragg peaks 


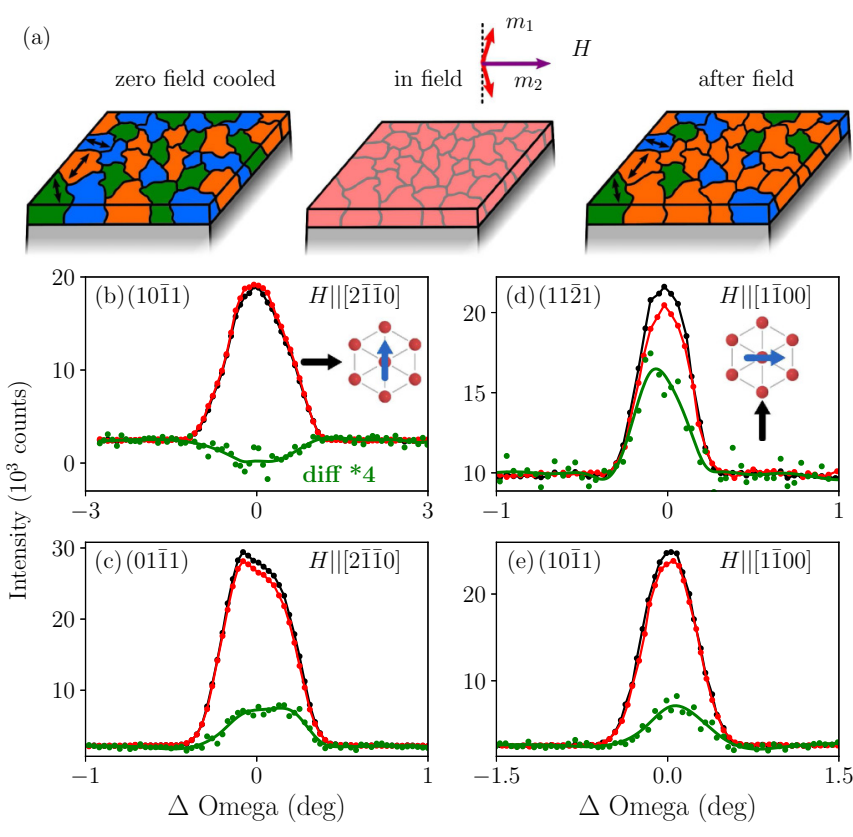

FIG. 5. (a) Effect of a strong magnetic field on the domain population in MnTe thin films. After zero-field cooling, the population of the three distinct magnetic domains is equal. When a strong field, i.e., above the spin-flop field, is applied, the canted magnetic moments (see inset) of all domains align almost perpendicular to the magnetic field. After releasing the field, a higher population of the domain with the easy axis close to perpendicular to the field direction remains (orange domains in the sketch). (b)-(e) Neutron diffraction curves recorded at a temperature of $5 \mathrm{~K}$ after zero-field cooling (black) and after application and removal of a magnetic field of $6 \mathrm{~T}$ (red). The difference between the black and red curves is shown multiplied by a factor of 4 in green. The effect of the magnetic field is shown for two field directions differing by $90^{\circ}$. Insets in (b) and (d) show to orientation of the magnetic field (blue arrow) within the $c$ plane and indicate the direction of the primary neutron beam (black arrow).

can therefore be used to determine the magnetic moment of the $\mathrm{Mn}$ atoms. By comparison with simulations using the FULLPROF SUITE [39], we find a magnetic moment between 4.7 and $5 \mu_{B}$ at low temperature, which is in agreement with earlier studies [13,15].

Such intensity ratios, however, cannot be directly used to determine the in-plane orientation of the magnetic moments. When the sample is cooled in zero magnetic field, magnetic domains equally populate the various equivalent crystallographic directions [7,9] [cf. Fig. 5(a)] and the sample appears to be isotropic in the $c$ plane. To break this symmetry, one can apply a strong in-plane magnetic field above the spin-flop transition, to enforce domain repopulation [7]. Such a field forces the moments in an orientation nearly perpendicular to the applied magnetic field and therefore the magnetic diffraction intensities also do not contain the desired information about the in-plane easy axis. However, when the strong applied field is removed, the domains with easy axis direction closest to perpendicular to the field direction are preferentially populated. For the case when an in-plane field is applied perpendicular to one of the easy axis, this means that domains with this Néel vector orientation will be preferred
TABLE II. Relative change of the absolute value of the structure factor after the application of a $6 \mathrm{~T}$ field in the specified direction. The respective experimental data are shown in Fig. 5. The simulated change for easy axes along $\langle 1 \overline{1} 00\rangle$ and $\langle 2 \overline{1} \overline{1} 0\rangle$ is listed and the former simulation (highlighted) agrees within error bars with experimental data.

\begin{tabular}{lcrlc}
\hline \hline peak & $H$ direction & $\Delta\left|F_{\mathrm{EXP}}\right|(\%)$ & $\Delta\left|F_{\text {SIM }}^{\langle\langle\mathbf{1} \mathbf{1 0 0}\rangle}\right|$ & $\Delta\left|F_{\mathrm{SIM}}^{\langle 2 \overline{1} \overline{1} 0\rangle}\right|$ \\
\hline$(10 \overline{1} 1)$ & {$[2 \overline{1} \overline{1} 0]$} & $1.27 \pm 0.16$ & $\mathbf{1 . 3 0 \%}$ & $1.30 \%$ \\
$(01 \overline{1} 1)$ & {$[2 \overline{1} \overline{1} 0]$} & $-2.60 \pm 0.08$ & $\mathbf{- 2 . 6 0}$ & -2.60 \\
$(11 \overline{2} 1)$ & {$[1 \overline{1} 00]$} & $-7.23 \pm 0.30$ & $\mathbf{- 7 . 2 4}$ & -7.21 \\
$(10 \overline{1} 1)$ & {$[1 \overline{1} 00]$} & $-2.11 \pm 0.08$ & $\mathbf{- 2 . 0 3}$ & -1.22 \\
\hline \hline
\end{tabular}

over the two other domains with Néel vector orientation tilted by $30^{\circ}$ with respect to the field direction. From the difference of the domain repopulations for various magnetic field directions, one can determine the easy axes directions. Below we show that a magnetic field of $6 \mathrm{~T}$ is sufficient to repopulate the domains since it triggers the spin-flop transition. Neutron diffraction measurements before (black) and after (red) the application of a magnetic field for various Bragg peaks and two field directions are shown in Figs. 5(b)-5(e). As magnetic field directions we use the high-symmetry directions within the

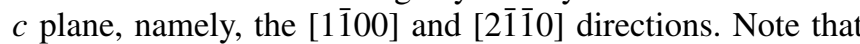
the measurements before and after application of a magnetic field were performed on the very same sample, which first was

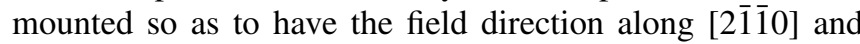
removed after the measurement of Figs. 5(b) and 5(c), heated above Néel temperature and remounted to have the field along [1100] to measure Figs. 5(d) and 5(e). The difference between the measurements before and after the field, i.e., the signal corresponding to the remnant domain repopulation, are shown as the green curves. It is this difference which will be further quantified and analyzed in Table II.

Structure factors $F$ were extracted from the measurements using the software COLL5 [40], which considers geometrical effects from the measurement setup, resulting in different full width at half maximum values for different Bragg reflections shown in Fig. 5. The relative difference of structure factors before and after the application of magnetic field $H: \Delta\left|F_{\text {EXP }}\right|=\left(\left|F_{\text {EXP }}^{\text {after }}\right|-\left|F_{\text {EXP }}^{\text {before }} \mathrm{H}_{\mid}\right|\right) /\left|F_{\text {EXP }}^{\text {before }}\right|$ is listed in Table II for selected Bragg peaks and [2110] and [1100] field directions. In order to derive the easy axis direction, we further modeled the structure factors with FULLPROF for two different easy axes directions. As potential easy axis directions we consider two high symmetry directions: the $\langle 1 \overline{1} 00\rangle$ direction indicated in Fig. 1(a) and the direction perpendicular to it in-plane, i.e., $\langle 2 \overline{1} \overline{1} 0\rangle$. To derive the simulated change of the structure factor $\Delta\left|F_{\mathrm{SIM}}\right|$, we additionally model the efficiency of the domain repopulation after the application of a 6-T magnetic field.

As mentioned above, the magnetic field leads to higher population of the domain(s) with easy axis closer to the field normal. Since the efficiency of this process is unknown, we considered it a free parameter in our model. The domain population is described by three occupation numbers, which add up to unity. Each occupation number corresponds to the occupation of a domain with Néel vector orientation along one of the three crystallographically equivalent axis within the $c$ 
plane. Taking into account the field directions and considered easy axis directions, this means that we either equally favor or disfavor two sets of domains. This means that one parameter is sufficient to describe the domain repopulation in either case. Since the two different field directions with respect to the easy axes directions likely result in different domain repopulation efficiencies, this means we have two free parameters in the model. Within this model, the observed changes of the structure factors $\Delta\left|F_{\mathrm{EXP}}\right|$ in Table II can only be consistently explained when we consider the easy axes to be along the $\langle 1 \overline{1} 00\rangle$ directions (cf. $\Delta\left|F_{\text {SIM }}^{\langle 1 \overline{1} 00\rangle}\right|$ in Table II). The two free parameters describing the domain population thereby result in populations of $\sim 40 \%: 30 \%: 30 \%$ and $\sim 39 \%: 39 \%: 22 \%$ for the three distinct easy axes directions after the application of the field perpendicular and parallel to one easy axis. In Fig. 5(a), the change of the domain population by the application of a field perpendicular to an easy axis, which leads to the increase of one population, and corresponding decrease of the population of the two other domains is qualitatively sketched. In agreement to Ref. [7], a single domain state is unachievable at least after removal of the magnetic field. The determined easy axes are consistent with the susceptibility data measured by SQUID [cf. Fig. 3(c)], which found the lowest susceptibility at low temperature when the field is aligned along the [1100] direction, or any other equivalent direction.

\section{MAGNETOTRANSPORT}

Since thick enough films for neutron diffraction cannot be obtained for MnTe on $\operatorname{InP}(111)$ we employed an alternative approach to determine the easy axis directions in this case. Using the crystalline contribution [41] to the anisotropic magnetoresistance (AMR), the easy axis can also be determined. Radial flow of electrical current in Corbino disks suppresses the noncrystalline components [42] and the remaining crystalline contribution $\propto \cos (6 \phi)$ due to the hexagonal symmetry of the material serves as a straightforward detector of the Néel vector direction. Here the angle $\phi$ corresponds to the angle between the Néel vector and the easy axis direction. Corbino contacts, sketched in the inset of Fig. 6(a), were fabricated on MnTe thin layers (50-nm thick) grown on InP by depositing gold contact rings using a lithographic lift-off process.

During an in-plane rotation of applied magnetic field, also when its strength is above the spin-flop threshold, the anisotropy makes the Néel vector lag behind the direction perpendicular to the field when the former is located near an easy axis. Consequently, deviations from the $\cos (6 \phi)$ form can be observed in Fig. 6. This means that, as soon as the Néel order can be influenced by external magnetic field, the easy axis can be determined from such transport measurements. In Fig. 6(a), we show the field direction (specified by the angle $\psi$ ) dependence of the longitudinal resistance for magnetic fields up to $10 \mathrm{~T}$. While at low fields almost no effect of a field rotation is observed, a dominantly six-fold signal arises in stronger fields. Figure 6(b) shows the variation of the $\cos (6 \psi)$ contribution to the AMR signal for different field strengths. The mentioned contribution shows a clear onset just below $2 \mathrm{~T}$ and saturates for fields above $6 \mathrm{~T}$, indicating that all moments rotate slightly canted aligned almost perpendicular to the stronger fields. We note that the sixfold variation of
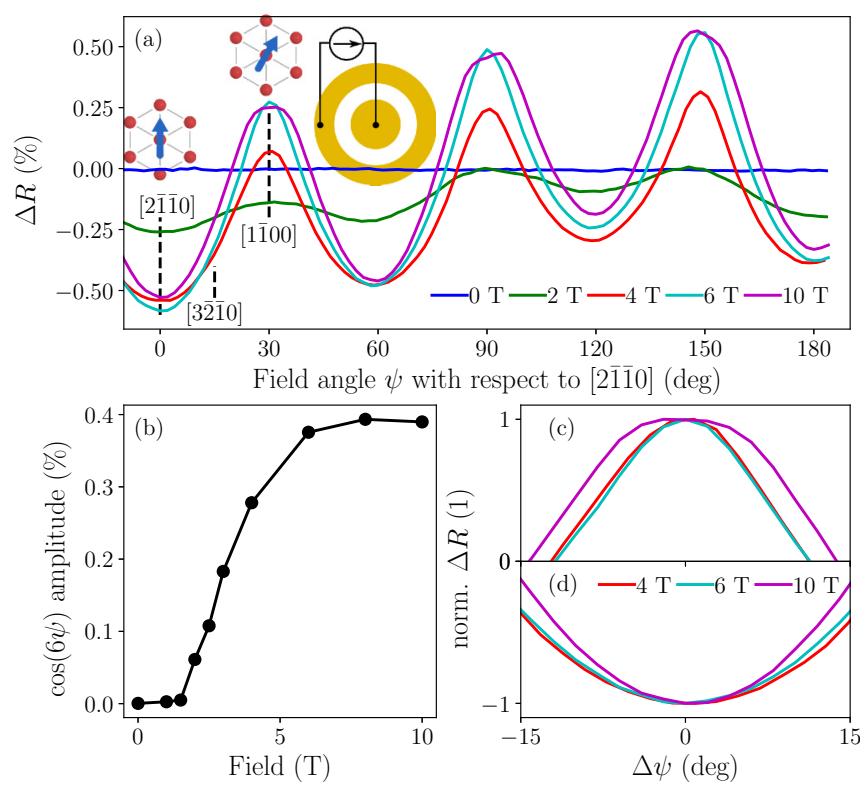

FIG. 6. (a) Longitudinal resistance traces during magnetic field rotations in $50 \mathrm{~nm} \mathrm{MnTe}$ on $\mathrm{InP}(111)$ for different field strengths. An inset shows the Corbino disk measurement geometry, and three magnetic field directions are marked by their crystallographic directions. (b) Variation of the amplitude of the dominant $\cos (6 \phi)$ contribution to the resistance change. The amplitude was determined using a Fourier decomposition of the measured resistance change. (c) and (d) Zooms to the minimum and maximum resistance values during the field rotations. Maxima in (c) are narrower than minima in $(\mathrm{d})$.

the resistance shows clear differences between the maxima and minima of the resistance variation. As it is visible in Figs. 6(c) and 6(d), the minima always appear wider than the maxima. This indicates that magnetic moments are pushed towards the position of the minima in the resistance by the in-plane anisotropy. Considering that the magnetic field is nearly perpendicular to the moments we infer that the easy axes are oriented along $\langle 1 \overline{1} 00\rangle$. Note that the difference between minima and maxima is decreasing in stronger fields as the MA is becoming smaller relative to the external magnetic field.

\section{SPIN-FLOP FIELD MEASUREMENTS}

In Fig. 7, we plot magnetic field dependent measurements, revealing the spin-flop transition detected by various methods using both considered substrate materials. Figure 7(a) shows the magnetization per Mn atom measured by a SQUID magnetometer when strong magnetic fields are applied. As expected for an antiferromagnet, the magnetization of the sample is mostly compensated and only a fraction of Bohr magneton $\mu_{B}$ is detected even above the spin-flop transition. When the field is applied in the out-of-plane $c$ direction, a featureless linear trace is observed, while for in-plane field small changes of the slope appear (best visible in the inset), indicating the spin-flop transition. Since our system comprises multiple domains and three in-plane easy axis directions the traces deviate from the more common spin-flop signals in uniaxial antiferromagnets $[43,44]$. However, the characteristic features with smaller 

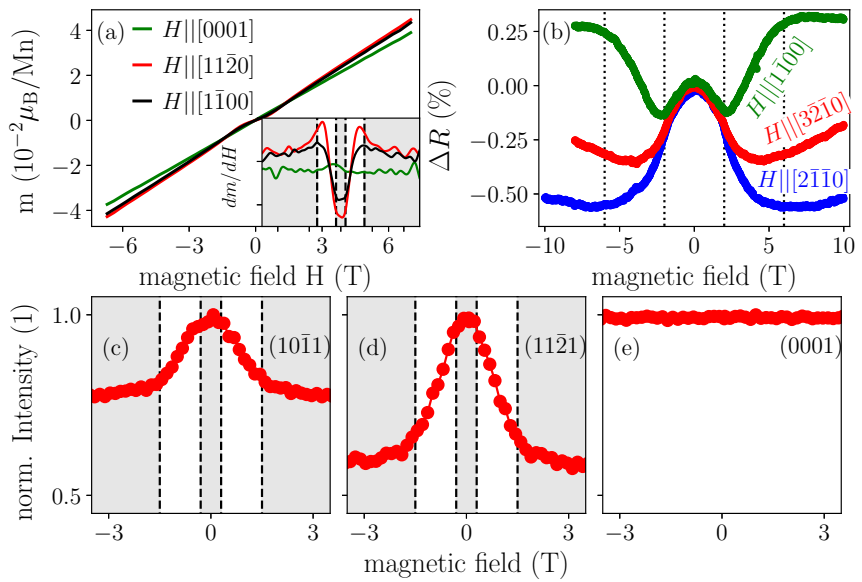

FIG. 7. Magnetic field sweeps performed using different methods. (a) Magnetic moment per $\mathrm{Mn}$ atom of $\mathrm{MnTe}$ on $\mathrm{SrF}_{2}$ determined by SQUID for magnetic fields applied in various directions. The diamagnetic contribution of the substrate was subtracted. An inset shows the derivative of the magnetic moment by the magnetic field. For the in-plane measurements distinct regions are detected which are distinguished by the slope of $m(T)$. (b) Change of the longitudinal resistance in $\mathrm{MnTe}$ on $\mathrm{InP}(111)$ measured in Corbino disk geometry vs magnetic field applied along the [2 $\overline{1} \overline{1} 0]$, [3 $\overline{2} \overline{1} 0]$, and [11̄00] directions. (c)-(e) Normalized neutron diffraction intensity

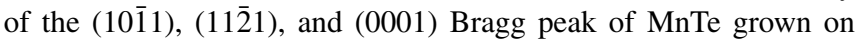
$\mathrm{SrF}_{2}$ during a magnetic field sweep with field along the [1]100] direction.

slope below the spin-flop field and a higher slope above the spin-flop field are clearly visible in our data. Note that as expected for an antiferromagnetic material, the slope of the traces at high fields when extrapolated to zero crosses through zero, which excludes any ferromagnetic contribution. The net magnetization of $\sim 0.04 \mu_{\mathrm{B}} / \mathrm{Mn}$ at the highest field of $6 \mathrm{~T}$ corresponds to a canting angle smaller than $1^{\circ}$.

Field dependent neutron diffraction intensities shown in panels Figs. 7(c)-7(e) confirm the spin-flop field as observed by the SQUID magnetometer. Similar to the SQUID measurements, different regions in Figs. 7(c) and 7(d) can be identified (indicated by gray background color). At small fields (below $0.5 \mathrm{~T}$ ) and above $\sim 1.5 \mathrm{~T}$, the intensities are rather constant while up to $40 \%$ changes are observed between 0.5 and $1.5 \mathrm{~T}$. This shows that a certain field needs to be overcome to start the reorientation of the moments. Once the reorientation is complete no changes occur in the neutron diffraction intensities since in contrast to SQUID neutrons are not sensitive enough to detect the small magnetic moment induced by the canting of the two magnetic sublattices. It is important to note that the magnetic diffraction peak (0001) is unaffected because the magnetic moments remain in the basal plane and therefore are always perpendicular to the momentum transfer. This again confirms that the [0001] direction is the hard axis of the system in agreement with our theoretic predictions and magnetic susceptibility measurements in Fig. 3.

Magnetic field sweeps in transport measurements shown in Fig. 7(b) also show significant changes associated with the spin flop. Instead of the reorientation of moments between $\sim 0.5$ and $1.5 \mathrm{~T}$, as seen by neutron diffraction and SQUID, the onset of AMR in these measurements is located between $\sim 2$ and $6 \mathrm{~T}$. This large change implies that MA in both samples are different since the spin-flop field is proportional to square root of the MAE [45]. Note that the sample used in these transport studies was grown on $\mathrm{InP}(111)$ which causes different strain. Although the easy axis directions determined from neutron diffraction for films grown on $\mathrm{SrF}_{2}$ are found to be the same as the ones determined from AMR for films on InP, the strength of the in-plane anisotropy is different. On InP the tensile strain in MnTe at low temperature (mostly due to thermal expansion coefficient mismatch) causes a bigger magnetic anisotropy, resulting in a higher spin-flop field for those samples.

\section{CONCLUSION}

Our neutron diffraction, magnetometry, and magnetotransport measurements in combination with $\mathrm{DFT}+U$ calculations confirm that antiferromagnetic NiAs-type MnTe thin layers are magnetically an easy plane material. Within the hexagonal basal plane, the magnetic anisotropy is considerably smaller than the out-of-plane anisotropy and moreover, can be engineered by choosing suitable substrate and working temperature. For MnTe films on $\mathrm{InP}$ and $\mathrm{SrF}_{2}$ at low temperatures, the easy axis is [1100] (or any of the other two crystallographically equivalent directions). The strain induced by the thermal expansion coefficient mismatch on InP causes tensile strain within the $c$ plane and results in significantly higher spin-flop fields. Onsets of the spin-flop transition change from $\sim 0.5 \mathrm{~T}$ for films grown on $\mathrm{SrF}_{2}$ to $\sim 2 \mathrm{~T}$ for films grown on InP. The moderate spin-flop field allows to repopulate magnetic domains even in the antiferromagnetic state, which was exploited to determine the easy axis direction from neutron diffraction. The small in-plane anisotropy opens up the possibility to vary the resistance of the material almost continuously due to the AMR effect [7]. This together with its simple collinear magnetic structure makes MnTe a favorable model system to test antiferromagnetic spintronics phenomena.

\section{ACKNOWLEDGMENTS}

We acknowledge support from the Austrian Science fund (J-3523-N27), the Grant Agency of the Czech Republic (grant No. 14-37427G), ERDF (project "Nanomaterials center for advanced applications," CZ.02.1.01/0.0/0.0/15_003/0000485), the Ministry of Education of the Czech Republic Grant NoS. LM2015087 and LNSM-LNSpin, the EU FET Open RIA Grant No. 766566, and the ERC Synergy Grant No. 610115.
[1] T. Jungwirth, X. Marti, P. Wadley, and J. Wunderlich, Nat. Nanotechnol. 11, 231 (2016).
[2] V. Baltz, A. Manchon, M. Tsoi, T. Moriyama, T. Ono, and Y. Tserkovnyak, arXiv:1606.04284. 
[3] K. Olejník, V. Schuler, X. Marti, V. Novák, Z. Kašpar, P. Wadley, R. P. Campion, K. W. Edmonds, B. L. Gallagher, J. Garces, M. Baumgartner, P. Gambardella, and T. Jungwirth, Nat. Commun. 8, 15434 (2017).

[4] X. Marti, I. Fina, C. Frontera, J. Liu, P. Wadley, Q. He, R. J. Paull, J. D. Clarkson, J. Kudrnovský, I. Turek, J. Kuneš, D. Yi, J.-H. Chu, C. T. Nelson, L. You, E. Arenholz, S. Salahuddin, J. Fontcuberta, T. Jungwirth, and R. Ramesh, Nat. Mater. 13, 367 (2014).

[5] H. Reichlová, D. Kriegner, V. Holý, K. Olejník, V. Novák, M. Yamada, K. Miura, S. Ogawa, H. Takahashi, T. Jungwirth, and J. Wunderlich, Phys. Rev. B 92, 165424 (2015).

[6] P. Wadley, B. Howells, J. Železný, C. Andrews, V. Hills, R. P. Campion, V. Novák, K. Olejník, F. Maccherozzi, S. S. Dhesi, S. Y. Martin, T. Wagner, J. Wunderlich, F. Freimuth, Y. Mokrousov, J. Kuneš, J. S. Chauhan, M. J. Grzybowski, A. W. Rushforth, K. W. Edmonds, B. L. Gallagher, and T. Jungwirth, Science 351, 587 (2016).

[7] D. Kriegner, K. Výborný, K. Olejník, H. Reichlová, V. Novák, X. Marti, J. Gazquez, V. Saidl, P. Němec, V. V. Volobuev, G. Springholz, V. Holý, and T. Jungwirth, Nat. Commun. 7, 11623 (2016).

[8] S. Onari, T. Arai, and K. Kudo, J. Phys. Soc. Jpn. 37, 1585 (1974).

[9] T. Komatsubara, M. Murakami, and E. Hirahara, J. Phys. Soc. Jpn. 18, 356 (1963).

[10] Non-Tetrahedrally Bonded Binary Compounds II, edited by O. Madelung, U. Rössler, and M. Schulz, Landolt-BörnsteinGroup III Condensed Matter Vol. 41D (Springer-Verlag, Berlin, Heidelberg, 2000), p. 1, Chap. MnTe: crystal structure, physical properties.

[11] C. Ferrer-Roca, A. Segura, C. Reig, and V. Muñoz, Phys. Rev. B 61, 13679 (2000).

[12] J. D. Wasscher, Ph.D. thesis, Technische Hogeschool Eindhoven, 1969.

[13] N. Kunitomi, Y. Hamaguchi, and S. Anzai, J. Physique 25, 568 (1964).

[14] J. B. C. Efrem D'Sa, P. A. Bhobe, K. R. Priolkar, A. Das, S. K. Paranjpe, R. B. Prabhu, and P. R. Sarode, J. Magn. Magn. Mater. 285, 267 (2005).

[15] W. Szuszkiewicz, B. Hennion, B. Witkowska, E. Usakowska, and A. Mycielski, Phys. Status Solidi C 2, 1141 (2005).

[16] E. Przezdziecka, E. Dynowska, W. Paszkowicz, W. Dobrowolski, H. Kepa, C. Majkrzak, T. Giebultowicz, E. Janik, and J. Kossut, Thin Solid Films 516, 4813 (2008).

[17] W. Szuszkiewicz, E. Dynowska, B. Witkowska, and B. Hennion, Phys. Rev. B 73, 104403 (2006).

[18] L. Chua, IEEE Trans. Circuit Theory 18, 507 (1971).

[19] P. Meuffels and R. Soni, arXiv:1207.7319.

[20] S. Lequeux, J. Sampaio, V. Cros, K. Yakushiji, A. Fukushima, R. Matsumoto, H. Kubota, S. Yuasa, and J. Grollier, Sci. Rep. 6, 31510 (2016).
[21] K. Ando, K. Takahashi, T. Okuda, and M. Umehara, Phys. Rev. B 46, 12289 (1992).

[22] H. Akinaga, K. Ando, T. Abe, and S. Yoshida, J. Appl. Phys. 74, 746 (1993).

[23] E. Janik, E. Dynowska, J. Bak-Misiuk, M. Leszczyhki, W. Szuszkiewicz, T. Wojtowicz, G. Karczewski, A. K. Zakrzewski, and J. J. Kossut, Thin Solid Films 267, 74 (1995).

[24] B. Hennion, W. Szuszkiewicz, E. Dynowska, E. Janik, and T. Wojtowicz, Phys. Rev. B 66, 224426 (2002).

[25] W. Kim, I. J. Park, H. J. Kim, W. Lee, S. J. Kim, and C. S. Kim, IEEE Trans. Magn. 45, 2424 (2009).

[26] Z. Wang, D. Geng, J. Li, Y. Li, and Z. Zhang, J. Mater. Sci. Technol. 30, 103 (2014).

[27] Z. Wang, D. Geng, W. Gong, J. Li, Y. Li, and Z. Zhang, Thin Solid Films 522, 175 (2012).

[28] J. Zemen, J. Kučera, K. Olejník, and T. Jungwirth, Phys. Rev. B 80, 155203 (2009).

[29] A. B. Shick and W. E. Pickett, Phys. Rev. Lett. 86, 300 (2001).

[30] E. Wimmer, H. Krakauer, M. Weinert, and A. J. Freeman, Phys. Rev. B 24, 864 (1981).

[31] V. P. Antropov, V. N. Antonov, L. V. Bekenov, A. Kutepov, and G. Kotliar, Phys. Rev. B 90, 054404 (2014).

[32] P. Novák, DIPAN (Dipolar anisotropies), http://susi.theochem. tuwien.ac.at/reg_user/textbooks/usersguide.pdf, accessed 10/2017.

[33] S. Greenwald, Acta Cryst. 6, 396 (1953).

[34] G. Kommichau, H. Neumann, W. Schmitz, and B. Schumann, Cryst. Res. Technol. 21, 1583 (1986).

[35] R. Minikayev, E. Dynowska, B. Witkowska, A. M. T. Bell, and W. Szuszkiewicz, X-Ray Spectrom. 44, 394 (2015).

[36] V. M. Glazov, K. Davletov, A. Ya. Nashel'skii, and M. M. Mamedov, Zh. Fiz. Khim. 51, 2558 (1977).

[37] T. Nagamiya, K. Yosida, and R. Kubo, Adv. Phys. 4, 1 (1955).

[38] K. Chen, A. M. Ferrenberg, and D. P. Landau, Phys. Rev. B 48, 3249 (1993).

[39] J. Rodríguez-Carvajal, Physica B 192, 55 (1993).

[40] M. S. Lehmann and F. K. Larsen, Acta Cryst. 30, 580 (1974).

[41] E. DeRanieri, A. W. Rushforth, K. Výborný, U. Rana, E. Ahmad, R. P. Campion, C. T. Foxon, B. L. Gallagher, A. C. Irvine, J. Wunderlich, and T. Jungwirth, New J. Phys. 10, 065003 (2008).

[42] A. W. Rushforth, K. Výborný, C. S. King, K. W. Edmonds, R. P. Campion, C. T. Foxon, J. Wunderlich, A. C. Irvine, P. Vašek, V. Novák, K. Olejník, J. Sinova, T. Jungwirth, and B. L. Gallagher, Phys. Rev. Lett. 99, 147207 (2007).

[43] I. S. Jacobs, J. Appl. Phys. 32, S61 (1961).

[44] Y. S. Oh, S. Artyukhin, J. J. Yang, V. Zapf, J. W. Kim, D. Vanderbilt, and S.-W. Cheong, Nat. Commun. 5, 3201 (2014).

[45] T. Bernstein, Am. J. Phys. 39, 832 (1971). 